\title{
Desarrollo de una escala para medir las áreas sensible, racional y moral en estudiantes universitarios
}

\author{
Marsela Alejandra Álvarez Izazaga \\ Instituto Nacional de Ciencias Médicas y Nutrición Salvador Zubirán, México \\ José Antonio Roldán Amaro \\ Instituto Nacional de Ciencias Médicas y Nutrición Salvador Zubirán, México \\ Lucy María Reidl Martínez \\ Universidad Nacional Autónoma de México
}

Recibido: 15 de abril de 2015 / Aprobado: 25 de junio de 2015

El objetivo del estudio fue evaluar la efectividad de la Escala Tridimensional de la Toma de Decisiones en la Vida Cotidiana (ETDVC) para medir las características conductuales en tres áreas: sensible, racional y moral. La muestra estuvo conformada por 646 estudiantes de las carreras de Filosofía, Teología, Ciencias y Artes Plásticas en universidades de la Ciudad de México, de los cuales 261 son mujeres y 385 hombres, la edad promedio fue de 22,5 años. El instrumento final está conformado por 69 reactivos. La ETDVC presenta evidencias de validez y confiabilidad para medir las características desde la perspectiva tridimensional.

validez / confiabilidad / áreas sensible, racional y ética / toma de decisiones

\section{Development of a Three-Dimensional Scale to Measure Sensitive, Rational and Moral Areas in University Students}

The objective of this study was to evaluate the effectiveness of Decision Making in Daily Life (ETDVC) a three-dimensional scale for measuring behavioral characteristics in three areas: sensible, rational and moral. The sample consisted of 646 students majoring in philosophy, theology, science and arts in universities in Mexico City. The average age was 22.5 years; 260 females, 384 males were tested using the 69-item instrument. We conclude that ETDVC is valid and reliable for measuring characteristics from the threedimensional perspective.

validity / reliability / sensitive rational and moral areas / decision making

Correo electrónico: lucym@unam.mx 


\section{INTRODUCCIÓN}

Tan antiguas y tan vigentes como el hombre, tan polémicas y sencillas, así resultan las teorías que pretenden explicar el comportamiento desde una perspectiva tridimensional. Fueron los griegos - por medio de una de las grandes escuelas filosóficas del helenismo denominada estoicismo, fundada alrededor del año 300 a. C. por Zenón de Citio-quienes manejaron muy probablemente por primera vez la trilogía humana en términos de cuerpo, mente y espíritu (Asimov, 1973).

En uno de sus famosos Diálogos, Platón (1922) recurre al mito del "Tiro alado" para explicar la conducta humana. Se refiere al carro griego con un par de caballos con alas y conducido por un auriga. Decía que los mortales son como un carro conducido por un caballo noble y otro instintivo, lo cual hace difícil la conducción. El ser pensante es el auriga o piloto del carro griego y los dos caballos que lo jalaban representaban lo pasional y espiritual. De esa forma ambos corceles tiraban pretendiendo tener el control del carro, pero el hombre o conductor podía y debía guiar con la rienda los caballos donde su juicio decidiera.

Más tarde, en la filosofía cristiana, san Agustín de Hipona (354-430) fue quien elaboró por primera vez una explicación sistemática de la conducta humana desde una perspectiva trinitaria. Sin embargo, tuvieron que pasar quince siglos para rescatar su pensamiento, como humanista, teólogo y filósofo. Agustín concibió al ser humano con alma, valores, un código moral y principios; además, como un ente con capacidad de pensar, discutir y transformar; $y$ finalmente a un ser humano instintivo y pasional, que necesitaba ser feliz, amar y ser amado (San Agustín, 1948).

En el contexto psicológico, las teorías psicoanalíticas son las que más se apegan a lo que se planteará en este trabajo.

Freud (1993) advirtió la necesidad de crear una teoría para hacer comprensible su conceptualización del Yo. Elaboró una topografía hipotética del aparato psíquico en la que se estudian tres sistemas que denominó instancias psíquicas, las cuales interactúan dinámicamente, y que dividió en Yo, Superyó y Ello; cada una con características determinadas, según su influencia en la actividad psicológica.

El Ello se refiere al conjunto de impulsos instintivos del individuo, necesidades biológicas, deseos y motivaciones afectivas primarias, que bajo el principio del placer buscan su realización inmediata, presionando al Yo, que opera con el sentido de realidad para obtener una gratificación urgente.

El Superyó es el resultado de la incorporación dentro del Yo de los mandatos prohibitivos de los padres, es la internalización de la compulsión externa. Metafóricamente hablando se puede decir que ve y oye internamente, adquiriendo una autoridad sobre el Yo. Tiene, a su vez, el 
poder de realizar o no el acto prohibido, según sea su capacidad de resistencia. También se encarga de infligir castigo o culpa a través de su presencia permanente en la conducta humana. El término designa la instancia que en la personalidad normal modifica e inhibe automáticamente los impulsos instintivos del Ello, que tienden a producir acciones y pensamientos antisociales o inmorales. Es, por tanto, una especie de conciencia moral con sentido dinámico. Finalmente, opera bajo el principio del deber.

El Yo, ateniéndose al principio de realidad, trata de ajustar las pulsiones del Ello (dominado por el principio de placer) a las exigencias del Superyó (dominado por el deber moral). Su papel es interceder entre los impulsos y deseos instintivos, por un lado y las presiones morales, por el otro, fuerzas a menudo inconscientes, y entre estas y las exigencias del medio social. Más recientemente, algunos autores (Kubie, 1951; Tallaferro, 1983) plantean que el Yo no solo es el resultado pasivo de las influencias energéticas internas o externas, sino una estructura en virtud de la cual el hombre ha de hacerse cargo de la situación para subsistir. Esta estructura, a su vez, tiene su base en la corteza cerebral que es la que hace al hombre estar en su realidad. Es decir, el concepto primario e hipotético de Freud se habría modificado sobre bases puramente psicológicas, que tendría ahora una base neuropsicológica que correspondería a lo que él intuyó en sus investigaciones
(Tallaferro, 1983), como se comprobará más adelante, con la explicación del cerebro trino de McLean (1973) y su enfoque neurofisiológico.

Eric Berne (1961) es otro exponente importante de las teorías plurinominales, que explican la conducta humana. Menciona que cada individuo tiene a su disposición un repertorio limitado de estados del Yo, que no son representaciones sino realidades psicológicas:

- Los que semejan a las figuras paternas (exteropsíquicos, Yo padre).

- Los que están independientemente dirigidos hacia la apreciación objetiva de la realidad (neopsíquicos o Yo adulto).

- Los que representan reliquias arcaicas del Yo todavía activos, los cuales fueron fijados desde la infancia (arqueopsíquicos o Yo niño).

Por lo tanto, plantea que cada individuo:

a) Ha tenido padres (o tutores) y lleva en su interior una serie de datos del Yo que reproducen los estados de ánimo de estos padres (tal como él los vivió), y que esos estados del Yo paternales pueden ser activados en ciertas circunstancias.

b) Es capaz de pensar objetivamente si el estado del Yo apropiado puede ser activado.

c) Fue más joven de lo que ahora es y lleva en su interior fijaciones de sus primeros años de vida que pueden ser activadas en ciertas circunstancias. 
En este contexto surge el diagrama estructural que representa un esquema gráfico y completo del individuo. Incluye tres estados del Yo: el paternal, el adulto y el infantil.

El estado paternal se presenta en dos formas: directa e indirecta; como un estado del Yo activo y como influencia. Cuando es directamente activo, la persona responde como su propio padre (o madre) lo hacía. Cuando es una influencia, el individuo responde en la forma en que los padres querían que respondiera aunque ellos no lo hicieran. En el primer caso él se convierte en uno de ellos; en el segundo, se adapta simplemente a los requerimientos.

El estado adulto es necesario para la supervivencia. Es el que piensa objetivamente y calcula las probabilidades que son esenciales para tratar efectivamente con un mundo adverso.

El estado infantil es más biológico e imparcial y en realidad es un niño en muchos aspectos, la parte más valiosa de la personalidad, que puede contribuir a la vida del individuo, exactamente como un niño verdadero suele hacerlo en la vida familiar. El niño se muestra en dos facetas: el niño adaptado y el niño natural. El primero es el que modifica su comportamiento bajo la influencia paterna; esta es la causa y el niño adaptado el efecto. El niño natural es una expresión espontánea, es rebelde y creativo; es intuitivo, impulsivo y busca el placer.
Víctor Frankl (1977), fundador de la Tercera Escuela Vienesa de Psicoterapia, llamada logoterapia, es otro de los autores que aporta una teoría más en el contexto tridimensional. Plantea que el hombre no solo se halla dominado por una impulsividad inconsciente, como descubre su maestro Freud, sino también por una espiritualidad inconsciente.

Para Frankl (1994), el ser humano es una unidad tridimensional, "una totalidad corpóreo-anímico-espiritual". Enfatiza: nunca podremos insistir demasiado en que esta triple totalidad es lo que constituye al hombre entero. Así pues, de ningún modo está justificado hablar del hombre solamente como una totalidad psico-física, como lo ha sido hasta ahora por la comunidad estudiosa. Cuerpo y mente pueden bien formar una unidad psicofísica, pero nunca podría dicha unidad representar la totalidad humana. Sería una visión parcial del hombre, porque lo espiritual, que se excluye en esa dualidad, le pertenece como lo más esencial.

Asimismo, en el análisis existencial Frankl identifica en el ser humano tres importantes dimensiones: somática, psíquica y noética (llamada también como del logo o del espíritu) (Cueli, Reidl, Martí, Lartigue y Michaca, 2002).

El análisis existencial de Frankl señala que la existencia noética es un modo específico del ser, cuya característica de fondo radica en que no es un ser fáctico, sino facultativo. Lo que 
constituye a la persona espiritual como tal es su capacidad de tomar actitud ante sí misma, o si lo decide, tomar distancia de su dimensión psicofísica. Solo así es cómo el ser humano se estructura a sí mismo como una unidad espiritual y psicosomática.

McLean (1973) postuló el enfoque neurofisiológico y el cerebro trino. Elaboró un modelo que explica la estructura y evolución cerebral. Al cerebro de formación más antigua lo denomina el complejo reptílico o complejo $\mathrm{R}$, cuyo origen se remonta a varios centenares de millones de años. Rodeándolo se encuentra el segundo cerebro o sistema límbico, llamado así porque limita con el cerebro propiamente dicho, seguramente se formó hace más de 150 millones de años. Por último, el tercer cerebro se encuentra en el neocortex o corteza cerebral, sin duda la estructura más evolucionada en el hombre.

El complejo R sigue desempeñando dentro del cerebro humano las mismas funciones que cumplían los reptiles prehistóricos. Juega además un papel importante en la conducta agresiva y la territorialidad. Produce pautas de comportamiento muy similares a los reptiles que matan por instinto aunque de manera planeada.

La filósofa Susanne Langer (1942), escribió:

la vida humana está punteada de actos instintivos, como son los hábitos de los animales. La conducta humana es una obra intrincada en la que se entremezclan la razón y el rito; el saber y la religión; la prosa y la poesía; la realidad y los ensueños. El ritual como el arte son en esencia la culminación activa de una transformación simbólica de la experiencia, se engendra en la corteza, no en el "cerebro primitivo", pero es fruto de una necesidad elemental de dicho órgano una vez desarrollado este, hasta acceder al estadío humano actual. (Citada en Sagan, 1980, p. 80)

El sistema límbico contiene las raíces del comportamiento altruista así como la preservación de la especie a través de la reproducción, cuyo fin principal es la supervivencia. Muchas facetas del comportamiento de los animales tienden a refrendar la noción de que las emociones intensas en aras de la supervivencia son básicamente privativas de los mamíferos, y en menor medida de las aves. En los mamíferos se puede encontrar un comportamiento peculiar en favor de la comunidad o la supervivencia de la especie, no importando poner en riesgo la propia vida. Resta decir que las tradiciones heredadas a través de las generaciones se encuentran en esta área.

El neocórtex es la región donde se ubican las funciones cognitivas que definen al hombre como un ente racional. Esta porción de la corteza cerebral distingue cuatro regiones o lóbulos: frontal, parietal, occipital y temporal. Cada una de ellas regula multiplicidad de funciones, pero es probable que algunas sean 
ejercidas por más de un lóbulo a la vez. La mayoría de los neurofisiólogos han descubierto que el lóbulo frontal es el que nos permite anticiparnos para planear el futuro, lo que implica funciones cognoscitivas elaboradas. Se le atribuye también la capacidad de abstracción, el lenguaje simbólico, la lecto-escritura, el pensamiento lógico-matemático, el proceso de enseñanza aprendizaje.

En suma, la toma de decisiones de la vida cotidiana se da principalmente a través de la interacción dinámica de tres áreas: moral, racional y sensible. La explicación tridimensional de la conducta ya había sido desarrollada por Freud (1993) al hablar del aparato psíquico desde la perspectiva psicoanalítica. Plantea una hipótesis estructural que agrupa procesos y contenidos mentales conscientes e inconscientes que se relacionan funcionalmente $\mathrm{y}$ en el que distingue diferentes estructuras mentales en una interacción permanente y cualitativa que reside en tres instancias: el Ello, el Yo y el Superyó. El Ello, que abarca las interacciones psíquicas de las pulsiones y opera desde la óptica del principio del placer; el Yo, que consiste en aquellas funciones que tienen que ver con la relación en el medio social y operan bajo la óptica del principio de realidad, y el Superyó, que comprende los preceptos morales de nuestra mente, así como nuestras aspiraciones ideales, y operan bajo la óptica de los principios morales.

Antes de Freud, san Agustín (1948) también había explicado el compor- tamiento humano desde la óptica del hombre trinitario, es decir un ser humano que actúa desde la interrelación de: a) su moral y sus valores; b) su capacidad de discernir y pensar, y c) sus sentimientos y pasiones.

Berne (1982), por su parte, lo explica con su diagrama estructural, que implica la dinámica conductual del individuo a través de lo que denomina transacciones. En él incluye los tres estados del Yo, que son: el paternal, el adulto y el infantil. El paternal, que representa al propio papá, educa y marca las reglas; el adulto, que es el que piensa objetivamente y razona, y el niño, que es el impulso creativo, sensible y espontáneo.

Finalmente, Viktor Frankl (1994) explica la conducta humana en los términos de una totalidad corpóreoanímica-espiritual, enfatizando que es esa triple totalidad la que constituye al hombre por entero: la unidad psicofísica y espiritual.

\section{CONCEPTOS BÁSICOS DE LA UNIDAD} TRIDIMENSIONAL EN LA TOMA DE DECISIONES

El enfoque que se plantea en este trabajo es la unidad tridimensional, integrada por tres áreas: sensible, racional y moral. Estas tres áreas contienen muchas de las características señaladas por los enfoques plurinominales explicados anteriormente. Sin embargo, también observan características propias, una autonomía y desarrollo que se da al margen de las influencias paterna- 
les, familiares y sociales externas. Por ejemplo, los casos que rompen a veces radicalmente con el orden o principios establecidos, tanto en lo familiar como en lo social, como son los de los visionarios en lo artístico o en lo científico. Es decir, que lejos de repetir los esquemas de comportamiento y conocimiento aprendidos durante su infancia en el seno familiar, escolar o social, desarrollan ideologías o corrientes artísticas completamente ajenas a los modelos que los influenciaron o formaron; y que estas ideologías se ven reflejadas en las decisiones que toman diariamente de forma rutinaria, pero que muestran el predominio de alguna de las tres áreas.

El área sensible incluye todo aquello que es visceral, instintivo, espontáneo. Es el Yo niño en términos de Erick Berne (1982); emotivo, irracional, impulsivo, lo que Freud denomina el Ello. Es la parte pasional que no asume las reglas o normas para su comportamiento, simplemente siente, su meta es ser feliz, sentirse bien y lograr el placer. Actúa porque le gusta, lo desea, y en consecuencia rehúye a lo que no le place. Se rige por el principio del placer y su máxima expresión puede darse en el arte.

El área racional implica todo lo que es objetivo, racional, lógico. Es lo que Freud (1993) llama el principio de realidad y Berne (1982) denomina Yo adulto. Se desarrolla y funciona con capacidad argumentativa y analítica. Su máxima expresión puede darse en la ciencia.
El área moral se basa en los valores introyectados, los valores humanos, el respeto a la ley, a la autoridad, a las tradiciones; la religión. Berne (1982) la denominaría Yo padre y Freud (1993) como el Superyó y el principio del deber.

Cabe señalar que aunque existe una estructura general, esta es flexible y dialéctica, varía muchas veces en función particular de cada persona y situación específica. Podríamos afirmar que existen tantas variaciones como personas, pero lo hace sobre la base de la estructura elemental mencionada.

\section{HiPÓTESIS}

Los individuos desarrollan tres áreas en su personalidad: sensible, racional y moral, las mismas que observan dimensiones diferentes en el comportamiento; por ejemplo, donde solo una de ellas puede predominar. Dicha variabilidad es la que define las características de la conducta del individuo. La Escala Tridimensional de la Toma de Decisiones en la Vida Cotidiana (ETDVC) pretende medir tales dimensiones.

Se plantearon las siguientes preguntas:

1. ¿Es la escala ETDVC un instrumento eficaz para medir las características conductuales referentes a las áreas sensible, racional y moral de los estudiantes universitarios?

2. ¿Existen diferencias entre los estudiantes universitarios de áreas filosóficas, de ciencias y de arte en cuanto a la 
dimensión de sus áreas conductuales sensible, racional y moral?

\section{Objetivos}

\section{Objetivo general}

El objetivo general consiste en evaluar la efectividad de la ETDVC como un instrumento diagnóstico para medir las características conductuales en las áreas sensible, racional y moral y su dimensión (puntaje) en estudiantes universitarios.

\section{Objetivos específicos}

Los objetivos específicos son los siguientes:

1. Establecer la validez de constructo con grupos criterio de la ETDVC.

2. Estimar la confiabilidad de la ETDVC.

3. Identificar si los estudiantes de ciencias obtienen mayor puntaje que los demás en el área racional.

4. Establecer si los estudiantes de artes plásticas obtienen mayor puntaje en el área sensible que los demás.

5. Determinar si los estudiantes de filosofía/teología obtienen mayor puntaje en el área moral que los demás.

\section{Método}

Se realizó un estudio de tipo confirmatorio porque se pusieron a prueba las hipótesis estadísticas. Es de campo porque se acudió al lugar natural que rodeaba a los sujetos y transversal porque se estudió el fenómeno como sucedía en el presente y con una sola medición.

El diseño metodológico fue el cuasiexperimental, debido a que se está probando una técnica diagnóstica y no existe una manipulación de la variable independiente; además, los grupos de comparación no fueron formados al azar, sino que eran grupos ya establecidos, por lo que fue un diseño de tres grupos de comparación no equivalentes o también llamado diseño de tres grupos independientes.

\section{Participantes}

Se trabajó con una muestra total de 646 estudiantes del nivel de licenciatura, con una edad promedio de 22 años y medio, una edad mínima de 18 y una máxima de 41 años. El sexo de los estudiantes se distribuyó en 261 mujeres y 385 varones.

El tipo de muestreo fue no probabilístico intencional; para ello se trabajó con estudiantes del primero hasta el noveno semestres, predominando los alumnos de cuarto. Los sujetos fueron contactados en dos universidades: la Universidad Nacional Autónoma de México (UNAM) (471) y la Universidad Intercontinental (175). En la primera se acudió a la Facultad de Ciencias y a la Escuela Nacional de Artes Plásticas. En la segunda, a la escuela de Filosofía y Teología.

Debido a que la validez por grupos contrastados requiere de sujetos con 
características específicas que marquen diferencias entre sí y que estas se planteen en las hipótesis (Nunnally y Bernstein, 1998), se eligieron estudiantes de carreras con un perfil determinado que indicara el predominio de alguna de las áreas sensible, racional o moral. Se asumió que los estudiantes de artes plásticas cubrían el prototipo de las personas con un área sensible mayor que la racional o moral; los estudiantes de ciencias, personas con un área racional mayor que la moral y sensible, y los de teología y filosofía de la Universidad Intercontinental son estudiantes que pertenecen al seminario de los Misioneros de Guadalupe y cubrían el prototipo que debía tener una persona con el área moral más grande que la racional y sensible. Estos criterios, a su vez, fueron reafirmados por los requisitos que tenían que llenar los aspirantes para cada uno de los planteles de acuerdo al perfil vocacional de las carreras correspondientes. El total de sujetos por carrera se muestran en la tabla 1.

Tabla 1

Composición de la muestra por área y por carrera

\begin{tabular}{ccc}
\hline Área & Carrera & Núm. de sujetos \\
\hline Ciencias & Actuaría & 94 \\
& Biología & 13 \\
& Ciencias de la computación & 29 \\
& Física & 30 \\
Artes & Matemáticas & 21 \\
& Subtotal & 187 \\
& Artes visuales & 64 \\
& Diseño y Comunicación & 220 \\
Filosofía & Visual & $\mathbf{2 8 4}$ \\
& Subtotal & 115 \\
& Filosofía & 60 \\
Total & Teología & $\mathbf{1 7 5}$ \\
& Subtotal & 646 \\
\hline
\end{tabular}




\section{Instrumento}

La construcción, validación y confiabilidad de la ETDVC se llevó a cabo en 8 fases, que se describen con detalle en el capítulo de resultados. Aquí solo mencionaremos que se obtuvo como resultado un total de 69 reactivos estructurados como frases incompletas, cuyas opciones de respuesta son el complemento de la frase, y cada opción corresponde a un área, ya sea sensible, racional o moral.

\section{Procedimiento}

Para la aplicación del instrumento se solicitó la autorización de los directivos de las carreras de las universidades seleccionadas. Se acudió al director de la carrera de Filosofía y Teología de la Universidad Intercontinental, al de la Facultad de Ciencias y al de la Escuela Nacional de Artes Plásticas de la UNAM. Una vez que se obtuvieron las respectivas autorizaciones, se solicitaron los horarios y la ubicación de los salones de clases, para poder aplicar el instrumento.

Se acudió a los salones cuando los alumnos se encontraban en receso entre clase y clase; o en algunas ocasiones se le pidió al profesor en turno el tiempo para la aplicación del instrumento. Una vez frente a los alumnos, se les dieron las siguientes instrucciones:

Este es un cuestionario que pretende conocer su toma de decisiones sobre algunas cosas de la vida cotidiana, lo cual permitirá que conozcan su perfil vocacional. Contesten lo más since- ramente posible, cómo es que actúan generalmente ante las situaciones que se les exponen y no cómo les gustaría actuar o decidir. Muchas gracias.

Posteriormente se realizó el proceso de validación y confiabilidad del instrumento, el cual se describe con detalle en la siguiente sección.

\section{Resultados}

\section{Fase 1. Estudio piloto}

Se realizó por medio de un cuestionario con tres grupos de veinte personas a quienes se les explicó en qué consiste la teoría en la que se basa la ETDVC y las áreas que la componen (sensible, racional y moral). Posteriormente se les pidió que contestaran algunas preguntas referentes a cómo pensaban ellos que se comportaría, en diferentes aspectos de la vida cotidiana, una persona en la que predominara el área sensible; otra, el área racional; y la otra, el área moral.

\section{Fase 2. Construcción de reactivos}

Una vez que se concentraron los cuestionarios de los sesenta sujetos se analizaron para la construcción de los reactivos de la escala y se agruparon las respuestas en las diez actividades cotidianas que se muestran a continuación:

1. Forma de vestir

2. Forma de alimentarse

3. Forma de estudio

4. Forma de relacionarse con una persona amada 
5. Forma de divertirse

6. Forma de relacionarse con terceros

7. Forma de administrar el dinero

8. Forma de vivir los días conmemorativos

9. Forma de autopercibirse

10. Forma de relacionarse con la propia familia.

Se construyó un instrumento con cien reactivos, en el que cada uno presentaba tres opciones de respuesta y cada opción se referirá a una conducta del área sensible, racional y moral, respectivamente.

Es importante mencionar que cada reactivo estaba conformado por una afirmación incompleta y que se completaba con tres opciones de respuesta: una que se refería al área sensible, otra a la racional y otra a la moral. Los sujetos debían ordenar cada respuesta de acuerdo con la frecuencia en que realizaban cada conducta expuesta; el número 1 para la más frecuente, el 2 para la que hacía en término medio y el 3 para la menos usual. Así, cada opción de respuesta podía tener tres valores diferentes: 1, 2 o 3. Por esto para el análisis se tomaron de manera separada.

\section{Fase 3. Validez de contenido a través de jueces}

Los 100 reactivos que componían la ETDVC fueron sometidos al juicio de 21 psicólogos sociales y clínicos. Estos debían señalar: a) A cuál de los diez indicadores correspondía cada pregunta (véase la tabla 2), y

a) A qué área correspondía cada opción de respuesta: sensible, racional o moral.

Se contactó a cada juez por separado y se le indicó en qué consistía la evaluación que realizaría, se le explicó la teoría en la que se sustenta el instrumento y se le dio un ejemplo, con un reactivo diferente a los que iba a evaluar, de cómo debía contestar en cada reactivo con sus tres opciones de respuesta.

Con las evaluaciones de los 21 jueces se realizó un análisis de frecuencias para conocer el porcentaje de coincidencias y discrepancias entre los jueces y entre las dimensiones planteadas para la realización del instrumento.

El criterio de inclusión que se tomó para decidir si un reactivo era válido o no fue:

a) Que en los indicadores de los reactivos debería haber un porcentaje de coincidencias mayor al $50 \%$, porque son 10 posibilidades y el $10 \%$ se debe al azar.

b) Y en las tres opciones de respuesta referidas a las diversas áreas, el azar sería del $33 \%$, por lo que se duplicó el criterio y se decidió que las coincidencias debían ser del $66 \%$ o más.

Cada reactivo con sus tres opciones de respuesta debía cumplir con este criterio, si un reactivo no cumplía con este 
porcentaje mínimo, entonces quedaba fuera. Sin embargo, el promedio de coincidencia de los reactivos aprobados estuvo por encima del $75 \%$; y los que salieron fue por una diferencia muy pequeña al porcentaje de inclusión.

En la tabla 2 se puede observar que 69 reactivos tuvieron un alto porcentaje (de $90,1 \%$ a $100 \%$ ) de coincidencia entre los jueces y solo nueve no cumplieron con el porcentaje mínimo $(50,1 \%)$.
También se calculó una prueba de $\mathrm{X}^{2}$ para ver si las diferencias en las coincidencias eran significativas, en este análisis se encontró que solo en cuatro no hubo diferencias estadísticamente significativas (véase la tabla 3).

En cuanto a las opciones de respuesta, al ser 100 reactivos con tres opciones cada uno, los jueces evaluaron 300 opciones; 100 que correspondían al área sensible, 100 a la racional y 100 a la

\section{Tabla 2}

Número de reactivos en cada intervalo de porcentaje de coincidencia para cada indicador

\begin{tabular}{|c|c|c|c|c|c|c|}
\hline \multirow[b]{3}{*}{ Indicador } & \multicolumn{6}{|c|}{$\begin{array}{c}\text { Intervalos del } \% \text { de coincidencia } \\
\text { entre jueces }\end{array}$} \\
\hline & 0 a & 50,1 & 60,1 & 70,1 & 80,1 & 90,1 \\
\hline & 50 & a 60 & a 70 & a 80 & a 90 & a 100 \\
\hline Forma de vestir & 1 & 0 & 1 & 0 & 0 & 8 \\
\hline Forma de alimentarse & 0 & 0 & 1 & 1 & 0 & 8 \\
\hline Forma de estudiar & 3 & 1 & 0 & 0 & 3 & 3 \\
\hline $\begin{array}{l}\text { Forma de relacionarse con una } \\
\text { persona amada }\end{array}$ & 1 & 0 & 0 & 1 & 0 & 8 \\
\hline Forma de divertirse & 0 & 1 & 2 & 0 & 1 & 5 \\
\hline $\begin{array}{l}\text { Forma de relacionarse con } \\
\text { terceros }\end{array}$ & 1 & 0 & 0 & 2 & 1 & 6 \\
\hline Forma de administrar el dinero & 1 & 0 & 1 & 0 & 1 & 7 \\
\hline $\begin{array}{l}\text { Forma de vivir los días } \\
\text { conmemorativos }\end{array}$ & 1 & 0 & 0 & 0 & 2 & 7 \\
\hline Forma de autopercibirse & 1 & 0 & 0 & 0 & 1 & 8 \\
\hline $\begin{array}{l}\text { Forma de relacionarse con la } \\
\text { familia }\end{array}$ & 0 & 0 & 0 & 0 & 1 & 10 \\
\hline Total & 9 & 2 & 5 & 4 & 10 & 70 \\
\hline
\end{tabular}


moral. El criterio mínimo fue de $66 \%$ de coincidencia en cada una para que se quedara el reactivo. De estas 300 opciones solo 31 no cumplieron con el criterio y 269 sí cumplieron.
De esta manera, al observar los puntos de coincidencia entre los indicadores y las opciones de respuesta se obtiene un instrumento con 69 reactivos que sí cumplieron con los cuatro criterios que exigía esta fase.

Tabla 3

Análisis de las discrepancias y coincidencias de los jueces en las afirmaciones de los indicadores

\begin{tabular}{|c|c|c|c|c|c|c|c|c|}
\hline Reactivo & $X^{2}$ & p & Reactivo & $X^{2}$ & $P$ & Reactivo & $X^{2}$ & $p$ \\
\hline 1 & $a$ & & 35 & 13,76 & $<.0001$ & 69 & 13,76 & $<.0001$ \\
\hline 2 & 29,48 & $<.0001$ & 36 & 17,19 & $<.0001$ & 70 & 17,19 & $<.0001$ \\
\hline 3 & 10,71 & .001 & 37 & a & & 71 & 2,33 & .127 \\
\hline 4 & 17,19 & $<.0001$ & 38 & 14.23 & .003 & 72 & 30,86 & $<.0001$ \\
\hline 5 & a & & 39 & a & & 73 & a & \\
\hline 6 & 17,19 & $<.000$ & 40 & a & & 74 & 13,76 & $<.0001$ \\
\hline 7 & a & & 41 & a & & 75 & a & \\
\hline 8 & 41,29 & $<.0001$ & 42 & 8,00 & .018 & 76 & 17,19 & $<.0001$ \\
\hline 9 & 3,7 & .157 & 43 & 17,43 & $<.0001$ & 77 & a & \\
\hline 10 & a & & 44 & a & & 78 & 3,7 & .157 \\
\hline 11 & 17,19 & $<.0001$ & 45 & 14 & .001 & 79 & 14,86 & .001 \\
\hline 12 & a & & 46 & a & & 80 & 17,19 & $<.0001$ \\
\hline 13 & a & & 47 & 8,89 & .003 & 81 & a & \\
\hline 14 & 17,19 & $<.0001$ & 48 & 18 & $<.000$ & 82 & 17,19 & $<.0001$ \\
\hline 15 & 8,86 & .012 & 49 & 17,19 & $<.0001$ & 83 & a & \\
\hline 16 & 17,19 & $<.0001$ & 50 & a & & 84 & a & \\
\hline 17 & 8,05 & .005 & 51 & a & & 85 & $a$ & \\
\hline 18 & a & & 52 & 26 & $<.0001$ & 86 & 19,2 & $<.0001$ \\
\hline 19 & 0,048 & .827 & 53 & 30,85 & $<.0001$ & 87 & a & \\
\hline 20 & a & & 54 & 17,19 & $<.0001$ & 88 & 17,19 & $<.0001$ \\
\hline 21 & 17,19 & $<.0001$ & 55 & 10,29 & .006 & 89 & 13,76 & $<.0001$ \\
\hline 22 & 17,19 & $<.0001$ & 56 & 13,76 & $<.0001$ & 90 & a & \\
\hline 23 & 13,76 & $<.0001$ & 57 & a & & 91 & 17,19 & $<.0001$ \\
\hline 24 & a & & 58 & 8,05 & .005 & 92 & a & \\
\hline 25 & 17,19 & $<.0001$ & 59 & a & & 93 & 17,19 & $<.0001$ \\
\hline 26 & 10,29 & .006 & 60 & 17,19 & $<.0001$ & 94 & a & \\
\hline 27 & 26,00 & $<.0001$ & 61 & 26,95 & $<.0001$ & 95 & a & \\
\hline 28 & a & & 62 & a & & 96 & 29,23 & $<.0001$ \\
\hline 29 & 10,71 & .001 & 63 & 12,1 & .017 & 97 & 5,2 & .074 \\
\hline 30 & 30,85 & $<.0001$ & 64 & a & & 98 & 17,19 & $<.0001$ \\
\hline 31 & 17,19 & $<.0001$ & 65 & 2,33 & .127 & 99 & a & \\
\hline 32 & 21,42 & $<.0001$ & 66 & 3,86 & .050 & 100 & 17,19 & $<.0001$ \\
\hline 33 & $a$ & & 67 & $a$ & & & & \\
\hline 34 & 30,86 & $<.0001$ & 68 & 30,86 & $<.0001$ & & & \\
\hline
\end{tabular}

a No se calculó porque el $100 \%$ de los jueces coincidieron. 


\section{Fase 4. Recolección de datos}

Se aplicó el instrumento de 69 reactivos a la muestra antes descrita, en las dos universidades.

En todos los lugares donde se acudió hubo apoyo para que se aplicara la encuesta y tanto las autoridades como los estudiantes tuvieron la disposición y el interés.

Se recolectaron 646 cuestionarios contestados en su totalidad y se procedió a la captura y análisis de los datos por medio del paquete estadístico SPSSv23.00.

\section{Fase 5. Validez de constructo a través de grupos contrastados o grupos criterio (Anastasi y Urbina, 1998)}

Se sometieron los datos a un análisis de varianza para poner a prueba las hipótesis del estudio, las cuales plantean que los estudiantes de filosofía presentarían una media alta en el área moral; los de ciencias, una media alta en el área racional y los de artes en el área sensible. En la siguiente sección se describen estos resultados.

\section{Diferencias por rama profesional}

En cuanto a las comparaciones de los estudiantes se encontró que en el área moral del instrumento las tres ramas profesionales (ciencias, arte y filosofía) resultaron significativamente diferentes entre sí (de acuerdo con la prueba confirmatoria de Games-Howell). Los estudiantes de filosofía fueron los que tuvieron el puntaje más alto en esta área, les siguieron los de ciencias y finalmente los de artes (véase la tabla 5).

En el área racional se encontró que los alumnos de ciencias fueron los que presentaron mayor puntaje, enseguida los de arte, y los de filosofía fueron los que obtuvieron el puntaje más bajo. Sin embargo, de acuerdo con la prueba de Games-Howell, se comprueba que las diferencias significativas están dadas entre los estudiantes de filosofía contra los de artes y ciencias, siendo estos dos últimos iguales en su conducta racional (véase la tabla 5).

Finalmente, el área sensible del instrumento muestra que los estudiantes de artes presentaron la media más alta en esta área; les siguen los de ciencias, $\mathrm{y}$ estos difieren significativamente (de acuerdo con la prueba Games-Howell) de los estudiantes de filosofía, que fueron los que tuvieron la media más baja (véase la tabla 4).

En el interior de cada grupo se analizó si había diferencias significativas entre los puntajes que se obtuvieron en cada área; se encontró que en los estudiantes de ciencias las tres áreas son diferentes, predominando el área racional, seguida por la sensible y por último la moral (véase la tabla 5).

En los alumnos de artes se observa igualdad entre las áreas racional y sensible, pero estas difieren de manera 
Tabla 4

Comparación entre grupos con respecto a las áreas del instrumento

\begin{tabular}{lcccccc}
\hline \multicolumn{1}{c}{ Áreas } & Ciencias & Artes & Filosofía & $\mathbf{F}$ & $\mathbf{p}$ & $\eta^{2}$ \\
\hline Moral & 137,85 & 130,88 & 150,25 & 64,94 & $<.0001$ & \\
Racional & 145,5 & 144,5 & 136,61 & 16,49 & $<.0001$ & \\
Sensible & 142,03 & 144,48 & 130,52 & 38,29 & $<.0001$ & \\
\hline
\end{tabular}

Tabla 5

Comparación intragrupo en los estudiantes de las tres ramas profesionales

\begin{tabular}{lccccccc}
\hline Rama/Área & Moral & Racional & Sensible & $\mathbf{F}$ & $\mathbf{p}$ & $\mathbf{N}$ & $\eta^{2}$ \\
\hline Ciencias & $137,85^{*}$ & $145,66^{*}$ & $142,03^{*}$ & 14,08 & $<.0001$ & 187 & \\
Artes & $130,98^{*}$ & 144,14 & 144,44 & 83,16 & $<.0001$ & 284 & \\
Filosofía & $150,25^{*}$ & $136,63^{*}$ & $130,61^{*}$ & 81,9 & $<.0001$ & 175 & \\
\hline
\end{tabular}

* Indica qué grupo es diferente del resto.

significativa con el área moral, la que tuvo el puntaje más pequeño en comparación con las otras dos (véase la tabla 5).

Finalmente, en los alumnos de filosofía se encontraron diferencias estadísticamente significativas entre las tres áreas, donde el área moral fue la que tuvo el puntaje más alto, seguida del área racional, el más bajo fue para el área sensible (véase la tabla 5).

De acuerdo con estos resultados se observa que el instrumento sí es sensible para detectar las diferencias entre los grupos criterio, permitiendo así predecir cómo puntuaría cada grupo en las áreas racional, sensible y moral.

\section{Fase 6. Confiabilidad por mitades}

Para calcular la confiabilidad del instrumento se utilizó la técnica de división por mitades, para lo cual se dividió el instrumento en dos partes para cada área; en una estaban los reactivos pares y en la otra los nones. Posteriormente se correlacionaron las mitades de cada área y se aplicó la fórmula de Spearman-Brown:

$$
\mathrm{r}_{\mathrm{SB}}=\frac{2 r_{12}}{1+r_{12}}
$$

Donde:

$r_{12}=$ Correlación entre las dos mitades

$r_{\mathrm{SB}}=$ Correlación de Spearman-Brown 
Tabla 6

Índices de confiabilidad por área

\begin{tabular}{cccc}
\hline Área & $\mathbf{r}$ & $\mathbf{p}$ & $\mathbf{r}_{\mathrm{SB}}$ \\
\hline Sensible & .790 & $<.0001$ & .883 \\
Racional & .746 & $<.0001$ & .855 \\
Moral & .794 & $<.0001$ & .885 \\
\hline
\end{tabular}

Los índices de confiabilidad para cada área fueron bastante aceptables (véase la tabla 6).

\section{Fase 7. Confiabilidad por medio del coeficiente Alfa de Cronbach}

Se calculó el puntaje de Alfa de Cronbach para cada área, encontrándose puntajes altos. Para el área Moral fue de 0.8956, para el área Racional, de 0.8608, y para el área Sensible, 0.8856; lo cual implica alta consistencia interna en el instrumento y por lo tanto una alta confiabilidad en la medición.

\section{Comparación por sexo}

Se calcularon pruebas $t$ de Student para muestras independientes, para ver si el sexo era una variable que influía en las diferencias por área. Sí se encontraron diferencias significativas entre hombres y mujeres en las tres áreas.

Las mujeres presentaron una media mayor que los hombres en el área sensible y racional, y los hombres en el área moral $(\mathrm{p}<.005)$.

\section{Comparación por sexo y rama profesional}

Se realizó un análisis con la prueba $t$ de Student para hacer comparaciones por sexo en las tres ramas profesionales. Los resultados muestran diferencias interesantes. En los estudiantes de ciencias se encontró que las mujeres presentan un puntaje más alto que los hombres en el área moral y sensible $(\mathrm{p}<.05)$. Sin embargo, en el área racional los puntajes son iguales.

Al realizar un análisis en el interior de cada género, se encontró que en los hombres predomina el área racional sobre las áreas moral y sensible $(\mathrm{F}(2,646)=18.18, \mathrm{p}=.000)$. En cambio en las mujeres predominan dos áreas: la racional y la sensible sobre la moral $(\mathrm{F}(2,646)=3.16, \mathrm{p}=.048)$.

En los estudiantes de artes no se encontraron diferencias significativas entre hombres y mujeres en alguna de las áreas.

En cuanto a los estudiantes de filosofía sí se encontraron diferencias signi- 
ficativas: los hombres presentaron un puntaje más alto que las mujeres en el área moral $(\mathrm{t}=-2.06, \mathrm{p}<.05)$. En las mujeres no hubo predominio en alguna área.

Al analizar los puntajes de los hombres se observó que en estos predomina el área moral sobre la racional y esta sobre la sensible $(\mathrm{F}(2,646)=86.99 \mathrm{y}$ $\mathrm{p}<.0001$ ). En cambio, en las mujeres no se encontraron diferencias significativas entre las áreas, lo cual indica que las tres son del mismo tamaño ( $\mathrm{F}(2$, $646)=1.164 \mathrm{yp}=.384)$.

\section{DISCUSIÓN Y CONCLUSIONES}

De acuerdo con los resultados obtenidos en la presente investigación, y con base en la hipótesis general, se puede concluir que, efectivamente, la Escala Tridimensional de la Toma de Decisiones en la Vida Cotidiana (ETDVC) es un instrumento válido y confiable para medir las características conductuales que implican una toma de decisiones desde las dimensiones de tres áreas: sensible, racional y moral. Esto se comprueba con los resultados de la validez de contenido por medio de jueces, por la validez de constructo a través de grupos contrastados, por la confiabilidad por mitades y a través del Alfa de Cronbach.

Como se observa en los resultados, las coincidencias entre los jueces, -donde el contenido de los reactivos es coherente con lo que pretende medir-, son contundentes. En el $70 \%$ de los reactivos hay coincidencias que van del 90 al $100 \%$ de los jueces. Este tipo de validez es apoyada como efectiva por Nadelsticher (1983), Cronbach (1998) y Thorndike y Hagen (1975).

Por lo que respecta a la validez de constructo también apoya que el instrumento es capaz de distinguir grupos con diferentes características en las áreas sensible, racional y moral, tal como se esperaba en la segunda hipótesis de trabajo planteada, la cual menciona que los estudiantes de profesiones como filosofía, ciencias y artes serán diferentes en los puntajes del área sensible, racional y ética, respectivamente. La validez de constructo se tiene como una de las más adecuadas para la construcción de test psicológicos por la relación tan íntima que guarda con la teoría y con el método científico, ya que permite formular hipótesis que pueden ser comprobadas o refutadas en el proceso de validación (Anastasi y Urbina, 1998; Cronbach, 1998).

Para reforzar la validez del instrumento también se aplicó un análisis para calcular la convergencia y divergencia entre los diez indicadores de la ETDVC. Este método, también conocido como multirrasgo-multimétodo, fue una aportación hecha por Campbell y Fiske en 1959 (Anastasi y Urbina, 1998), quienes sugirieron que los resultados del mismo rasgo de personalidad medido a través de diferentes métodos deben convergir en un punto común, es decir, deben correlacionar alto, debido a que teóricamente están midiendo lo 
mismo. Para este trabajo se tomaron como rasgos las áreas sensible, racional y ética, y cómo métodos cada uno de los 10 indicadores de la prueba (véase Fase 2). Así que se calcularon las correlaciones de los diez indicadores en cada área (un rasgo diez métodos). Aunque las correlaciones de los indicadores en el interior de cada área (sensible, racional y moral) no eran mayores de .628, lo cual indica que fueron correlaciones medias y bajas, todas estadísticamente significativas; es decir, que los indicadores convergen y miden una variable con elementos comunes que justamente es lo que forma cada constructo (sensible, racional y moral).

Asimismo, esos autores afirman que los resultados obtenidos por el mismo método de medición empleado para dos o más rasgos diferentes, deben divergir o no deben correlacionar entre sí debido a que se supone son constructos diferentes los que se miden. Esto se apoya con los resultados obtenidos por las divergencias entre los resultados del mismo indicador (mismo método) en las diferentes áreas (diferentes rasgos). La divergencia se observa en las correlaciones tan bajas y hasta negativas que se encontraron en el mismo indicador entre las diferentes áreas.

En cuanto a la confiabilidad del instrumento a través de la técnica de mitades se observa que sí existe una correlación alta entre los reactivos pares y los nones, lo cual confirma lo establecido por Anastasi y Urbina (1998),
Thorndike y Hagen (1975) y Nunnally y Bernstein (1995).

Por lo tanto, el primer planteamiento o postulado que se establece en el trabajo donde se dice que los seres humanos tienen tres áreas principalmente: racional, moral y sensible, a partir de las cuales hay una interacción dinámica y dialéctica en la que se lleva a cabo la toma de decisiones, se comprueba claramente. El enfoque tridimensional, como se explicó ampliamente en el marco teórico, no es nuevo. Platón, hace más de dos mil años, en sus diálogos, concretamente en el diálogo de la metáfora de Fedro, explica la disyuntiva en la toma de decisiones desde una perspectiva trilateral. Tal metáfora es, sin duda, el primer ejemplo conocido de una toma de decisiones en un contexto tridimensional.

Más adelante, la filosofía cristiana -principalmente a través de San Agustín (1948) - desarrollará una nueva propuesta de la conducta trinitaria, donde el punto de partida son el cuerpo, la mente y el alma. Establece que el cuerpo y la mente son mortales, pero el alma es inmortal. El cuerpo tiende hacia el placer, lo material, lo mundano. La mente explica racionalmente las cosas. Y el alma, que es de Dios, enseña lo que se debe hacer. Por mucho tiempo se estableció un antagonismo entre el cuerpo, la mente y el alma, expresada en la frase de Platón retomada por los cristianos, el cuerpo es la cárcel del alma. Sin embargo San Agustín, en un acierto asombroso para su tiempo, 
plantea una dualidad complementaria entre lo mortal e inmortal, partiendo de la máxima bíblica: y creó Dios al hombre a imagen y semejanza. Por tanto, concluye que el hombre es trino y uno como Dios. Y desde esa perspectiva trinitaria explica su comportamiento.

En el campo de la psicología es Freud (1993) quien explica la conducta desde una relación dinámica de tres instancias, que dominó Yo, Superyó y Ello, y que corresponden de alguna manera con las áreas en cuestión, a través del principio del deber, el principio de la realidad y el principio del placer. En este mismo sentido, el psicólogo norteamericano E. Berne (1961), a través de su teoría de análisis transaccional o análisis conciliatorio, establece su propia dinámica trilateral, que llamó los tres estados del Yo: padre, adulto y niño.

En este contexto, los resultados de la investigación avalan que, efectivamente, la toma de decisiones en la conducta puede ser explicada desde la dinámica e interacción de tres áreas. En términos de este estudio se definen como: racional, moral y sensible, y son las mismas que mide satisfactoriamente la ETDVC.

Un hallazgo interesante lo encontramos en la Escuela Nacional de Artes Plásticas (ENAP). En principio, una de las hipótesis establecía que los alumnos de artes tendrían que calificar más alto en el área sensible, por encima de las otras dos áreas. Sin embargo, fue sorpresivo encontrar que si bien la media del ser sensible fue alta, también lo fue la media del área racional. La explicación a esto la encontramos al revisar el plan de estudios de la carrera de Diseño Gráfico y Comunicación Visual: las materias estaban cargadas hacia el manejo de programas de cómputo para diseño, el uso de la lógica y las matemáticas, lo cual significó un acierto en la sensibilidad del instrumento, que detectó tal orientación de la carrera aún cuando no estaba calculado. En otras palabras, podríamos decir que los alumnos de artes están estudiando una carrera fuertemente matizada por las ciencias exactas, idea que fue confirmada en una entrevista con el coordinador académico de la ENAP.

Al margen de los resultados, y apoyando la hipótesis de trabajo, en la etapa de la aplicación del instrumento se pudo observar características comunes en los estudiantes de la ENAP. Vestían de manera muy singular, con numerosos accesorios, como aretes, tatuajes, tintes en el cabello de diversos colores, playeras con diseños muy llamativos, maquillaje muy original a decir por ellas mismas, etc., lo cual indica un área sensible predominante, donde los cánones que marcan las convenciones sociales es lo menos importante para ellos. Otro detalle que se observó fue que en las paredes de los salones, muros y pasillos abundaban los grafitis con lemas diversos de protesta, las asistencias eran bajas y la llegada tarde era algo común.

De la misma forma, durante la aplicación de la ETDVC en la escuela de 
Teología y Filosofía se observó que la forma de convivir, de vestir y de conducirse en los alumnos, ponía de manifiesto los aspectos recatados, serios y discretos previstos en la hipótesis correspondiente, debido a su formación dentro del Seminario en la Universidad Intercontinental. Era evidente el respeto total hacia las reglas y la autoridad; las clases se iniciaban sin retraso, con prácticamente la totalidad de los alumnos en las aulas.

En la Facultad de Ciencias la experiencia fue similar, la vivencia en la aplicación confirmó también la hipótesis de que los criterios racionales son los más importantes, debido a que fueron los alumnos que más cuestionaron y preguntaron por la encuesta, y los que se organizaron prácticamente solos para su aplicación. Asimismo, su lenguaje resultaba ser directo, su vestir práctico y su interés evidente por tomar sus clases.

\section{Consideraciones finales}

En el presente estudio se deben considerar los siguientes aspectos:

No se pudo obtener una muestra representativa de estudiantes, porque se tuvo que limitar a los sujetos que se encontraban en ese momento en el salón de clases y que además accedieron a contestar el test.
Algunos instrumentos no fueron contestados en su totalidad o no se comprendió cuál era la forma de contestar y quedaron fuera del estudio.

En las carreras de Filosofía y Teología se encontraron muy pocas mujeres (7 en total), lo que provocó que en la comparación por sexo los grupos quedaran desbalanceados.

Se sugiere que la ETDVC se aplique en diferentes grupos sociales, por ejemplo en alumnos del último año de preparatoria, tomando como criterio de predicción la carrera que piensan estudiar. Entonces se vería si esta carrera se relaciona con las áreas sensible, racional o moral, y ver si la ETDVC funciona como predictor de la orientación vocacional. Por ejemplo, si un alumno menciona que desea estudiar la carrera de matemáticas deberá obtener puntajes más altos en el área racional que en las áreas sensible y moral.

Asimismo, el instrumento se puede aplicar, validar y confiabilizar en grupos donde la medición de las áreas sea un buen predictor de desempeño laboral.

Otra aplicación que se le puede dar a la ETDVC es el diagnóstico de la toma de decisiones tridimensional y favorecer el autoconocimiento de las personas interesadas en saber qué área o áreas predominan en su conducta cotidiana. 


\section{REFERENCIAS}

Anastasi, A., y Urbina S. (1998). Test psicológicos. (7. ed.). México, D. F.: Prentice Hall.

Asimov, I. (1973). Enciclopedia biográfica de ciencia y tecnología. Madrid: Alianza Diccionarios.

Berne, E. (1961). Transactional analisis in psychoterapy. New York: Grove Press, Inc.

Berne, E. (1982). Los juegos en que participamos. Psicología de las relaciones humanas. México, D. F.: Diana.

Cronbach Lee, J. (1998). Fundamentos de los test psicológicos. Madrid: Biblioteca Nueva.

Cueli, J., Reidl, L., Martí, C., Lartigue, T., y Michaca, P. (2002). Teorías de la personalidad. México, D. F.: Trillas.

Frank1, V. (1977). El inconsciente espiritual, la presencia ignorada de Dios. Barcelona: Herder.

Frankl, V. (1994). Ante el vacio existencial. Barcelona: Herder.

Freud, S. (1993). Obras completas. Buenos Aires: Amorrortu Editores.

Kubie, L. (1951). Modern concepts of the organization of the brain. The Psychoanalytic Quarterly, 20.

Langer, S. K. (1958). Nueva clave de la filosofía. Un estudio acerca del sim- bolismo de la razón, del rito y del arte. Buenos Aires. Recuperado de http://www.filosoficas.unam.mx/ catalogo/?autores=langer-susanne-k

McLean, P. D. (1973). A triune concept of the Brain and Behaviour. Toronto: University of Toronto, Press.

Nadelsticher, M. A. (1983). Técnicas para la construcción de cuestionarios de actitudes y opción múltiple. México: Instituto Nacional de Ciencias Penales.

Nunnally, J. C., y Bernstein, I. J. (1998). Teoría psicométrica. (3. ${ }^{\mathrm{a}}$ ed.). México, D. F.: McGraw-Hill.

Platón (1922). Diálogos de Platón. México, D. F.: SEP-UNAM.

Sagan, C. (1980). Los dragones del Edén: reflexiones sobre la evolución de la inteligencia humana. Barcelona: Grijalbo.

San Agustín, santo obispo de Hipona. (1948). La Trinidad (De Trinitate). L. Arias, O.S.A. (trad.). Madrid: Biblioteca de Autores Cristianos.

Tallaferro, A. (1983). Curso básico de psicoanálisis. México, D. F.: Paidós.

Thorndike, R. L., y Hagen, E. (1975). Test y técnicas de medición en psicología y educación. México, D. F.: Trillas. 
